\title{
EFEITO DO ESFORÇO FÍSICO NO DESEMPENHO DE TIRO DE POLICIAIS MILITARES DO BATALHÃO DE CHOQUE
}

EFFECT OF PHYSICAL EFFORT ON SHOOTING PERFORMANCE OF MILITARY OFFICERS OF THE RIOT POLICE

EFECTO DEL ESFUERZO FÍSICO EN LA EJECUCIÓN DEL TIRO DE LOS POLICÍAS MILITARES DEL BATALLÓN ANTIDISTURBIOS

Luiz Inácio do Nascimento Neto ${ }^{1,2}$ (Profissional de Educação Física)

Daniel Gomes da Silva Machado 1,2 (Profissional de Educação Física) Pedro Moraes Dutra Agrícola ${ }^{2,3}$ (Profissional de Educação Física) Luiz Fernando de Farias Junior² (Profissional de Educação Física) André Igor Fonteles ${ }^{2,4}$ (Profissional de Educação Física) Samara Karla Anselmo da Silva² (Profissional de Educação Física) Hassan Mohamed Elsangedy² (Profissional de Educação Física) Alexandre Hideki Okano² (Profissional de Educação Física)

1. Universidade Estadual de Londrina, Centro de Educação Física e Esporte, Londrina, PR, Brasil.

2. Universidade Federal do Rio Grande do Norte, Departamento de Educação Física, Natal, RN, Brasil.

3. Faculdade Maurício de Nassau, Natal, RN, Brasil

4. Universidade de Fortaleza, Fortaleza, CE, Brasil

\section{Correspondência:}

Departamento de Educação Física, Universidade Federal do Rio Grande do Norte, Campus Universitário BR 101, Lagoa Nova, Natal, RN, Brasil. 59072-970. luizinacio50@gmail.com

\section{RESUMO}

Descritores: armas de fogo; fadiga; exercício; polícia; psicofisiologia.

\section{ABSTRACT}

Introdução: O uso de arma de fogo por policiais militares normalmente é precedido por perseguição a pé,e as alterações fisiológicas produzidas pelo esforço físico podem afetar o desempenho do tiro. Objetivo: Verificar o efeito do esforço físico sobre o desempenho de tiro em policiais militares. Métodos: A amostra foi constituída por 15 homens ( $34,1 \pm 5,4$ anos; $\left.81,4 \pm 8,8 \mathrm{~kg} ; 171,3 \pm 5,6 \mathrm{~cm} ; 27,7 \pm 2,3 \mathrm{~kg} / \mathrm{m}^{2} ; 44,9 \pm 4,0 \mathrm{ml}^{\mathrm{kg}} \mathrm{kg}^{-1} \cdot \mathrm{min}^{-1}\right)$ do batalhão de choque da polícia militar. Na primeira sessão, os participantes realizaram um teste de esforço máximo em esteira no qual se mediu o consumo pico de oxigênio $\left(\mathrm{VO}_{2 p i c o}\right)$. Na segunda sessão, foi avaliado o desempenho (pontuação) e tempo de tiro pré e pós-esforço físico e o tempo gasto no circuito de corrida com obstáculos (297 m). O índice de eficiência de tiro foi calculado pela razão entre a pontuação total e tempo de execução de tiro. O teste de Shapiro-Wilk confirmou a normalidade da distribuição dos dados. Utilizou-se o teste $t$ pareado para comparação das variáveis desfecho pré e pós-esforço e o coeficiente de correlação de Pearson. Resultados: O tempo para completar o percurso de corrida foi $75,3 \pm 4,4 \mathrm{~s}$. O desempenho $(20,4 \pm 11,4$ vs. $17,6 \pm 9,3$ pontos), tempo $(4,48 \pm 1,1$ vs. 4,23 $\pm 0,9$ s) e eficiência de tiro $(4,69 \pm 2,7$ vs. $4,35 \pm 2,7$ pts./s $)$ não apresentaram diferenças significativas $(P>0,2)$. Além disso, detectou-se uma correlação entre o $V_{2}$ pico e o tempo de execução da corrida $(r=-0,64 ; P=0,01)$, mas não com o desempenho de tiro $(P>0,05)$. Conclusão: $O$ esforço físico não afeta o desempenho de tiro de policiais militares. Apesar de o nível de aptidão aeróbica não ser associado ao desempenho de tiro, foi associado ao desempenho físico realizado previamente aos disparos.

Introduction: The use of firearms by military police officers is usually preceded by pursuit on foot, and the physiological changes produced by physical exertion may affect shooting performance. Objective: To verify the effect of physical effort on shooting performance in military police officers. Methods: The sample consisted of 15 men ( $34.1 \pm 5.4$ years; $\left.81.4 \pm 8.8 \mathrm{~kg} ; 171.3 \pm 5.6 \mathrm{~cm} ; 27.7 \pm 2.3 \mathrm{~kg} / \mathrm{m}^{2} ; 44.9 \pm 4.0 \mathrm{ml}^{\mathrm{kg}} \mathrm{kg}^{-1} \mathrm{~min}^{-1}\right)$ of the riot police. In the first session, the subjects performed a maximal treadmill stress test in which the peak oxygen consumption $\left(\mathrm{VO}_{2 \text { peak }}\right)$ was measured. In the second session, shooting performance (score) and shooting time were measured pre and post-exercise, as well as the time spent to complete a circuit of obstacle running $(297 \mathrm{~m})$. Shooting efficiency index was calculated as the ratio between total score and shooting time. The Shapiro-Wilk test confirmed the normal distribution of the data. A paired t-test was used to compare the post-exertion outcomes variables as well as the Pearson correlation coefficient. Results: The time to complete the running circuit was $75.3 \pm 4.4$ s. Shooting performance ( $20.4 \pm 11.4 \mathrm{vs} .17 .6 \pm 9.3$ points), time ( $4.48 \pm 1.1$ vs. $4.23 \pm 0.9 \mathrm{~s})$, and efficiency ( $4.69 \pm 2.7 \mathrm{vs} .4 .35 \pm 2.7 \mathrm{pts} / \mathrm{s})$ did not present significant differences $(P>0.2)$. Additionally, a correlation was detected between $V_{2}$ peak and running time $(r=-0.64 ; P=0.01)$, but not with shooting performance ( $P>0.05)$. Conclusion: Physical exertion does not affect shooting performance of military police officers. Although the level of aerobic fitness was not associated with shooting performance, it was associated with physical performance prior to shooting.

Keywords: firearms; fatigue; exercise; police; psychophysiology.

\section{RESUMEN}

Introducción: El uso de armas de fuego por los agentes de la policía militar suele ir precedido de persecución a pie, y los cambios fisiológicos inducidos por el esfuerzo físico pueden afectar el rendimiento de disparo. Objetivo: Determinar el efecto del esfuerzo físico en la ejecución del tiro de la policía militar. Métodos: La muestra consistió en 15 hombres $\left(34,1 \pm 5,4\right.$ años; $\left.81,4 \pm 8,8 \mathrm{~kg} ; 171,3 \pm 5,6 \mathrm{~cm} ; 27,7 \pm 23 \mathrm{~kg} / \mathrm{m}^{2} ; 44,9 \pm 4,0 \mathrm{ml} \cdot \mathrm{kg}^{-1} . \mathrm{min}^{-1}\right)$, del batallón antidisturbios de la policía militar. En la primera sesión, los participantes realizaron una prueba de esfuerzo máximo en una cinta rodante en la que se evaluó el pico de consumo de oxígeno $\left(\mathrm{VO}_{2 p i c o}\right)$. En la segunda sesión se evaluaron el rendimiento (puntuación) y el tiempo de disparo antes y después del esfuerzo físico y el tiempo trascurrido en el circuito de carrera con obstáculos (297 m). El índice de eficiencia de disparo se calculó como la relación entre la puntuación total y el tiempo de ejecución del tiro. La prueba de Shapiro-Wilk confirmó la distribución normal de los datos. Se utilizó la prueba t pareada para la comparación de las variables prey post-esfuerzo y el coeficiente de correlación de 
Pearson. Resultados: El tiempo para completar el curso de la carrera fue de $75,3 \pm 4,4$ s. El rendimiento $(20,4 \pm 11,4$ vs. $17,6 \pm 9,3$ puntos), el tiempo (4,48 $\pm 1,1$ vs. 4,23 $\pm 0,9$ s) y la eficiencia de disparo $(4,69 \pm 2,7$ vs. 4,35 $\pm 2,7$ pts. $/ \mathrm{s})$ no mostraron diferencias significativas $(P>0,2)$. Por otra parte, hemos detectado una correlación entre $V_{2 p i c o}$ y el tiempo para completar el circuito de la carrera $(r=-0,64 ; p=0,01)$, pero no con el rendimiento de disparo $(P>0,05)$. Conclusión: El esfuerzo físico no afecta el rendimiento de tiro en los policías militares. Aunque el nivel de la capacidad aeróbica no esté asociado con el rendimiento de tiro, se asoció con el rendimiento físico antes de disparar.

Descriptores: armas de fuego; fatiga; ejercicio; policía; psicofisiología.

\section{INTRODUÇÃO}

Atualmente, o tiro tem sido praticado de forma competitiva, lazer e também nas atividades policiais ${ }^{1,2}$. Logo, a situação e a forma como o tiro é realizado pode mudar drasticamente de acordo com a aplicação. Na esfera competitiva, o tiro esportivo, tem como objetivo acertar o maior número possíveis de disparos em um alvo ${ }^{3}$. Esses alvos podem ser fixos ou móveis e também podem variar quanto ao formato, distância e tamanho de acordo com a respectiva modalidade. Por outro lado, nas atividades policiais, o tiro, quando necessário, busca minimizar ao máximo o risco de lesões não intencionais aos civis inocentes e demais policiais ${ }^{1,4}$.

Dentre as atribuições dos policiais militares destacam-se a aplicação da lei intermediando conflitos sociais e prisão de suspeitos ${ }^{5}$. Assim, a atuação do policial militar é considerada de alto risco devido à exposição aos diversos fatores estressores nas situações de violência, tensão e morte envolvidas ${ }^{1,4,6}$. Adicionalmente, o emprego de arma de fogo por parte dos policiais militares geralmente é precedido por perseguições a pé, exigindo extrema habilidade de tiro ${ }^{7}$. Portanto, o militar deve estar preparado para executar o tiro com eficiência mesmo sob condições de stress físico e/ou psicológico, uma vez que sua sobrevivência e a dos demais envolvidos pode depender desta habilidade ${ }^{8}$.

O desempenho de tiro tem sido associado ao controle da respiração, concentração, ansiedade, frequência cardíaca, equilíbrio, força muscular e eficiência de gestos motores ${ }^{2,5,9-13}$. Contudo, durante confrontos armados a resposta fisiológica dos policiais desencadeia a reação de alarme do sistema nervoso simpático provocando o aumento da frequência respiratória, tremor, entorpecimento nas extremidades do corpo, limitação auditiva e do perímetro de visão, perda de destreza e da noção espaço-temporal ${ }^{14}$. Adicionalmente, os movimentos involuntários causados por tremores fisiológicos decorrentes da respiração, recuo balistocardíaco e pulsação arterial são fatores que comprometem o desempenho de tiro ${ }^{12}$. Portanto, é possível que as alterações fisiológicas decorrentes do esforço físico durante perseguições policiais afetem o desempenho de tiro.

Até o presente, poucos estudos analisaram a influência do esforço físico sobre o desempenho de tiro. Estudos realizados com atletas de elite da modalidade biatlo (esqui e tiro) evidenciaram uma piora no desempenho de tiro após o esforço físico ${ }^{15,16}$. Similarmente, militares do exército brasileiro apresentaram uma queda no desempenho de tiro após a corrida em uma pista com obstáculos ${ }^{17}$. Entretanto, o único estudo realizado com policiais militares não encontrou efeito do esforço físico sobre o desempenho de tiro $^{7}$. Contudo, tais estudos foram realizados com atletas ${ }^{15,16}$, em condições de esforço que não são representativas da atividade dos policiais militares, como por exemplo, exercício em ciclo ergômetro ${ }^{7}$, ou ainda com níveis de experiência de tiro restrita ${ }^{17}$.

Assim, torna-se importante conhecer o efeito do esforço físico em condições similares àquelas encontradas no cotidiano da atividade policial militar sobre o desempenho de tiro. Logo, considerando que a preparação dos militares quanto a acurácia e precisão de tiro para preservar a integridade física dos envolvidos é prioritária ${ }^{8}$, tais achados podem dar suporte para o desenvolvimento de programas de formação de policiais militares. Portanto, o objetivo deste estudo foi verificar o efeito do esforço físico realizado em pista de obstáculo adaptada para situação de perseguição sobre o desempenho de tiro de policiais militares. Nossa hipótese foi que o estresse fisiológico decorrente da realização do esforço em uma pista com obstáculos imediatamente antes de uma sessão de tiro perturbaria as variáveis fisiológicas afetando negativamente o desempenho de tiro.

\section{MÉTODOS}

A amostra final composta por 15 militares $(34,1 \pm 5,4$ anos; $81,4 \pm 8,8 \mathrm{~kg}$; $\left.171,3 \pm 5,6 \mathrm{~cm} ; 27,7 \pm 2,3 \mathrm{~kg} / \mathrm{m}^{2} ; 44,9 \pm 4,0 \mathrm{ml} \cdot \mathrm{kg}^{-1} \cdot \mathrm{min}^{-1}\right)$ do Batalhão de Choque da Polícia Militar todos com $\geq 6$ anos de experiência. Inicialmente, 18 militares do sexo masculino se voluntariaram para participar do presente estudo. Contudo, três participantes desistiram por incompatibilidade de horários. Os critérios de inclusão adotados foram estar inseridos no quadro do serviço ativo da Polícia Militar do Rio Grande do Norte, atuantes no policiamento ostensivo urbano e índice de massa corporal $<30 \mathrm{~kg} / \mathrm{m}^{2}$. Os critérios de exclusão foram: possuir qualquer problema osteomioarticular que impedisse a realização dos testes de esforço físico e uso de medicamento que alterasse a frequência cardíaca ou respiratória. O estudo foi aprovado pelo Comitê de Ética da Universidade Federal do Rio Grande do Norte (protocolo no: 752.339; CAAE: 31266114.3.0000.5537) e todos os participantes assinaram o termo de consentimento e livre esclarecido antes de serem admitidos no estudo.

O estudo foi realizado em duas sessões de avaliação em dias distintos com intervalo mínimo de 72 h entre as sessões. Na primeira sessão, foi realizada anamnese, aferição das medidas antropométricas, familiarização com a pistola de tiro utilizada no estudo e realização do teste incremental de esforço máximo para determinação da aptidão cardiorrespiratória. Na segunda sessão, os voluntários realizaram a sessão experimental. Primeiramente, realizaram uma série de disparos antes do esforço físico. Em seguida, após o último disparo, os indivíduos correram em um percurso com obstáculos que simula situações reais de perseguição policial. Imediatamente após finalizar o percurso, os sujeitos realizaram uma série de disparos após o esforço físico. Os sujeitos foram instruídos a evitar a ingestão de bebidas contendo cafeína e/ou álcool, a não realizar exercício físico nas 24 horas anteriores as sessões de avaliação. Todas as sessões de avaliação ocorreram no mesmo período do dia, entre 7 h00min e 9h00min.

As medidas antropométricas foram realizadas com os sujeitos descalços, vestindo o mínimo possível de roupa. A massa corporal em quilogramas foi aferida em uma balança digital (Welmy ${ }^{\circledR}$, W110H) e a estatura aferida em centímetros com estadiômetro acoplado na balança. Adicionalmente, o índice de massa corporal (IMC) foi calculado por meio da razão entre o peso e o quadrado da estatura.

O teste incremental de esforço máximo foi realizado em uma esteira rolante (Inbramed, ATL). Antes do teste, os participantes realizaram um aquecimento de cinco minutos com velocidade constante de $5 \mathrm{~km} / \mathrm{h}$ sem inclinação. O protocolo do teste incremental escalonado consistiu 
de velocidade inicial de $8 \mathrm{~km} \cdot \mathrm{h}^{-1}$ e incremento de $1 \mathrm{~km} \cdot \mathrm{h}^{-1}$ a cada minuto, e foi finalizado por exaustão voluntária. O teste foi considerado máximo quando a percepção subjetiva de esforço foi maior que 19 na escala de Borg ${ }^{17}$ (6-20) e/ou alcançar 95\% da frequência cardíaca máxima predita (220-idade). A frequência cardíaca foi monitorada durante todos os procedimentos de esforço físico por meio do monitor cardíaco (RS800cx, Polar Electro OU, Kempele, Finland).

\section{Análise da aptidão cardiorrespiratória}

A análise de troca gasosa foi realizada por um analisador de gases automático (Quark CPET, Cosmed, Roma, Itália) com sistema de análise respiração a respiração. O equipamento foi calibrado antes de cada teste de acordo as recomendações do fabricante. O consumo de oxigênio foi analisado em média de 20 segundos. 0 maior valor médio de 20 segundos durante o teste incremental foi considerado como consumo de oxigênio de pico $\left(\mathrm{VO}_{2 \text { pico }}\right)$.

\section{Testes de tiro}

No início da sessão os voluntários foram familiarizados com a pistola de pressão (Beeman ${ }^{\oplus} 2004$, calibre 4,5 mm, 0,77 kg), onde cada sujeito realizou três disparos. Os voluntários realizaram duas séries de cinco disparos uma antes e outra imediatamente após o esforço físico no alvo posicionado a uma distância de oito metros. Os disparos foram realizados na posição de pé, com empunhadura dupla, respeitando a individualidade de cada avaliado. Os alvos utilizados possuíam 15,5 cm de diâmetro e consistia de áreas zoneadas em círculos. O círculo no centro do alvo correspondia a dez pontos e possuía um centímetro de diâmetro. A partir do centro, os círculos aumentavam um centímetro de diâmetro e a pontuação diminuía em um ponto (Figura 1, esquerda). 0 alvo pontuado foi fixado em um suporte de 1,65 m de altura com uma figura humana impressa (Figura 1, direita).

Foram avaliadas (1) a pontuação total alcançada em cada série de disparos; (2) o tempo gasto para realização de cada disparo e (3) o índice de eficiência de tiro calculado como a razão da pontuação total obtida em cada série de disparos pelo tempo total gasto para realização dos disparos. O tempo de cada disparo foi registrado com cronometro digital por um único avaliador previamente treinado. O cronometro foi acionado imediatamente no momento em que o avaliado erguia os braços da posição ortostática em direção ao alvo para execução dos fundamentos de tiro e encerrado ao som do disparo. O teste de desempenho de tiro foi realizado no interior do Batalhão de Choque da Polícia Militar.

O circuito da pista de corrida com obstáculos foi utilizado na tentativa de simular situações cotidianas de perseguição realizadas por policiais militares. O percurso total da pista compreendeu uma distância de 297 m com cinco obstáculos distribuídos no percurso (Figura 2). O primeiro obstáculo consistia em subir e descer um aclive com solo

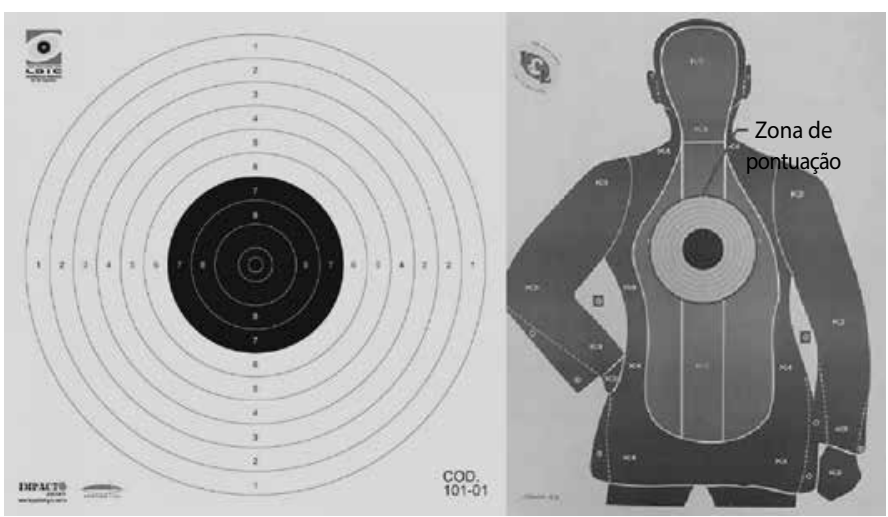

Figura 1. Alvo pontuado utilizado (esquerda) e figura humanoide (direita) onde o alvo era fixado na avaliação do desempenho antes e após o esforço físico. arenoso de aproximadamente 30 metros (15 m de aclive e $15 \mathrm{~m}$ de declive), similar a uma duna. Em seguida, se dirigiam em linha reta até o segundo obstáculo que consistia em entrar pela abertura de uma porta estreita em uma construção de um quarto e sair dela transpondo um muro de aproximadamente um metro de altura. No terceiro obstáculo, os voluntários realizaram uma corrida sinuosa (zigue-zague) entre três cones posicionados a um metro de distância um do outro. No quarto obstáculo os avaliados realizaram posição de tiro denominada de "torre" em quatro locais demarcados por cones com dois metros de distância entre eles, formando um losango. A posição de tiro "torre" consiste em o indivíduo ficar com um dos joelhos flexionados tocando o solo e o outro flexionado à frente dando apoio. No quinto obstáculo, os voluntários transpuseram novamente o primeiro obstáculo do circuito realizado em sentido oposto, terminando a corrida no mesmo ponto de início. Os voluntários foram instruídos a completar o circuito no menor tempo possível. Durante a realização do esforço foi dado forte encorajamento verbal. O tempo gasto para percorrer o circuito da pista com obstáculos foi cronometrado, sendo iniciado ao sinal de partida e finalizado no momento em que o avaliado cruzava a linha de partida. Para familiarização com o circuito da pista com obstáculos, previamente ao teste, os voluntários foram conduzidos por todo o percurso e orientados sobre o que deveriam cumprir em cada etapa.

\section{Analise estatística}

O teste de Shapiro Wilk confirmou a normalidade da distribuição dos dados. Os dados estão apresentados em média e desvio padrão. 0 teste $t$ de Student para amostras pareadas foi utilizado para comparar as variáveis de desempenho de tiro antes e após o esforço físico. 0 coeficiente de correlação de Pearson foi utilizado para analisar a correlação entre a aptidão cardiorrespiratória e as variáveis de desempenho de tiro. Foi adotado $p<0,05$ como significância estatística. Todas as análises foram realizadas no software SPSS 20.0. O poder estatístico do estudo foi calculado a posteriori usando o software Gpower (versão 3.1.9.2) resultando em um poder de $20 \%$ para as análises de comparação (Teste $t$ de Student para amostra pareadas) e de 79\% para as análises de correlação (Bivariate normal model).

\section{RESULTADOS}

Durante a corrida na pista com obstáculos os participantes atingiram uma intensidade de esforço de aproximadamente $85 \%$ da frequência cardíaca máxima alcançada no teste incremental máximo. O tempo

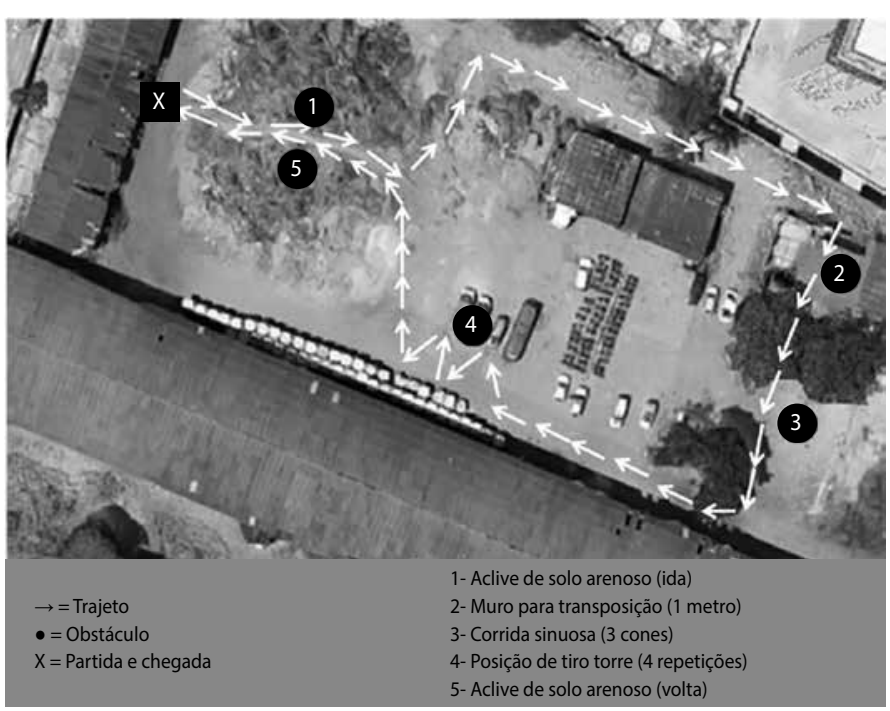

Figura 2. Visão por satélite do local de realização o esforço físico em pista de corrida com obstáculos. 
médio para percorrer o circuito da pista com obstáculos (297 m) foi de 75,3 \pm 4,4 segundos. A Figura 3 mostra a comparação do desempenho de tiro, tempo médio dos disparos e o índice de eficiência de tiro, nos momentos antes e após o esforço físico. Após a realização do esforço físico houve redução não significante de 13,73\% no desempenho de tiro $\left(\mathrm{t}_{(14)}=0,879 ; p=0,39\right)$, de $5,58 \%$ no tempo médio dos disparos $\left(t_{(14)}=1,231 ; p=0,24\right)$ e de 8,5\% no índice de eficiência de tiro $\left(\mathrm{t}_{(14)}=0,451 ; p=0,66\right)$.

A aptidão cardiorrespiratória apresentou correlação negativa com o tempo gasto para percorrer o circuito da pista com obstáculos $(r=-0,64 ; p=0,01)$. Entretanto, não houve correlação entre a aptidão cardiorrespiratória com o desempenho de tiro antes $(r=0,04 ; p=0,88)$ e após esforço físico ( $r=-0,46 ; p=0,08)$. Similarmente, não houve correlação entre a aptidão cardiorrespiratória com o tempo médio dos disparos antes ( $r=-0,29 ; p=0,30)$ ou após $(r=-0,26 ; p=0,35)$, assim como, com o índice de eficiência de tiro antes $(r=0,15 ; p=0,30)$ e após $(r=-0,27 ; p=0,34)$ esforço físico.

\section{DISCUSSÃO}

O presente estudo analisou o efeito do esforço físico sobre o desempenho de tiro em policiais militares do batalhão de choque. Interessantemente, nosso principal achado foi que o esforço físico realizado para percorrer uma pista com obstáculos, que simula situações de perseguição do cotidiano de policiais militares, não afetou o desempenho de tiro. Além disso, a aptidão cardiorrespiratória não apresentou correlação com nenhuma variável relacionada ao desempenho de tiro.

Apesar do prejuízo de 13,73\% nos valores de pontuação obtidos na série de disparos após o esforço físico comparado ao pré-esforço, não houve diferença estatisticamente significativa. Nossos resultados corroboram com outros estudos ${ }^{7,18,19}$. Brown et al. ${ }^{7}$ não encontraram efeito do esforço físico sobre o desempenho de tiro de policiais militares após uma sessão de exercício físico em ciclo ergômetro com intensidade 85\% do $\mathrm{VO}_{2 \text { pico. }}$ Contrariamente, estudos com atletas de biatlo e militares do exército brasileiro evidenciaram que o esforço físico piora o desempenho de tiro 8,15,16. Essa diferença nos resultados pode ser parcialmente explicada pela diferença nas armas utilizadas, pois enquanto os estudos que não verificaram efeito do esforço físico utilizaram pistola, os que o detectaram utilizaram rifle ou fuzil ${ }^{8,15,16}$. Nossa hipótese é que devido a diferença na forma de empunhadura o rifle pode ser mais susceptível a alterações fisiológicas como frequência cardíaca e pressão arterial pois, por ficar em contato com o tronco, armas longas podem sofrer maior influência das oscilações corporais. Portanto, acreditamos que o efeito do esforço físico sobre o desempenho de tiro parece depender também do tipo de arma utilizada.

Outra possível explicação para ausência de efeito do esforço físico sobre o desempenho de tiro é o bom nível de aptidão física dos indivíduos $\left(\mathrm{VO}_{2 \text { pico }}=44,9 \mathrm{ml} \cdot \mathrm{kg}^{-1} \cdot \mathrm{min}^{-1}\right)^{20}$. De acordo com Brown et al. ${ }^{7}$, as habilidades em realizar os fundamentos de tiro podem suprimir os efeitos fisiológicos prejudiciais ao desempenho de tiro. Logo, é possível que apesar das alterações fisiológicas decorrentes da demanda da corrida na pista com obstáculos que poderiam prejudicar o desempenho de tiro $8,12,14$, o estresse fisiológico tenha sido atenuado pelo condicionamento dos participantes.

Estudos recentes têm mostrado que apesar do aumento da frequência cardíaca, lactato sanguíneo e percepção de esforço o desempenho de tiro avaliado por diferentes parâmetros não é afetado ${ }^{18,19,21}$. Nibbeling et al. ${ }^{19}$ realizaram um estudo onde os participantes poderiam decidir quando correr ou atirar em um alvo em oito blocos de 150 segundos de esforço/tiro. A percepção subjetiva de esforço, nível de esforço mental e a frequência cardíaca aumentaram a cada bloco, atingindo no último bloco valor similar ao obtido em teste de esforço máximo. Os resultados mostraram que com maior nível de fadiga os participantes paravam de correr mais cedo, ficando a uma maior distância do alvo e, além disso, levaram mais tempo para atirar. Contudo, a acurácia permaneceu inalterada nos oito blocos ( 80\%). Logo, considerando que os participantes do referido estudo atiraram de uma maior distância e levaram mais tempo como forma de compensar a maior fadiga (maior PSE e FC) é possível que no presente estudo os policiais tenham controlado o nível de esforço despendido na pista com obstáculos, de modo a evitar possíveis efeitos negativos da corrida no desempenho de tiro, uma vez que a energia dependida no esforço pode ser decisiva no desfecho de uma situação.

Interessantemente, Evans et al.22 mostraram que exercícios fatigantes para membros superiores diminuiu o desempenho de tiro em soldados do exército. Os autores atribuem a piora na performance à fadiga dos músculos do tronco, que são responsáveis por estabilizar a parte superior do corpo e pode ter contribuído para o aumento na oscilação postural afetando o desempenho no tiro. Portanto, o efeito do esforço físico no desempenho de tiro parece também ser dependente do tipo de esforço realizado.

Similar ao resultado da pontuação total, o esforço físico prévio não alterou tempo de disparo, apesar da redução de 5,58\% (Figura 3B). Possivelmente, esse resultado é consequência do treinamento para execução do tiro durante os cursos de formação e aperfeiçoamento realizados pelos policiais militares, que os torna capazes de executar as técnicas básicas antes de realizar os disparos com a mesma precisão sem tempo adicional. Por outro lado, Dias et al. ${ }^{8}$ encontraram diminuição do tempo de execução dos disparos imediatamente após realização de exercício físico. A divergência dos resultados pode ser explicada pela característica das amostras. O estudo de Dias et al. ${ }^{8}$ foi realizado com militares recém-formados do exército brasileiro e, possivelmente, não tinham o nível de perícia completamente desenvolvido quanto militares mais experientes. Enquanto no presente estudo a amostra foi composta por policiais militares do batalhão de choque com pelos seis anos de experiência.

Outra medida de avaliação do desempenho de tiro analisada fo a eficiência de tiro. Esta medida reflete a eficiência do uso de arma de
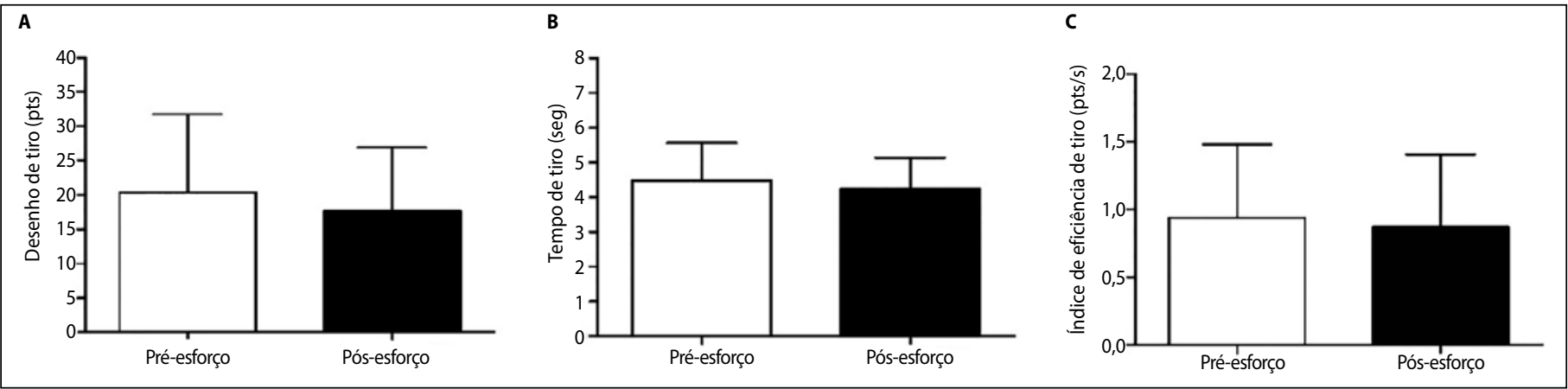

Figura 3. Desempenho, tempo e eficiência de tiro antes e imediatamente após a corrida em pista com obstáculos em policiais militares do batalhão de choque. 
fogo em ação rápida e precisa, buscando minimizar os riscos de prejuízos não intencionais em situações cotidianas dos policiais militares. Os resultados do presente estudo demonstram que não houve influência do esforço físico sobre o índice de eficiência de tiro (Figura 3C). Uma vez que não houve alteração no desempenho nem no tempo de tiro após esforço físico esse resultado era esperado.

Em relação às análises de correlação, houve apenas correlação negativa entre o nível de aptidão aeróbica e o tempo para percorrer o circuito da pista com obstáculos $(r=-0,64 ; p=0,01)$. Este resultado também era esperado, considerando que indivíduos com maior aptidão cardiorrespiratória apresentam maior desempenho em corrida e esforços físicos, cumprindo o circuito em menor tempo. Esse resultado corrobora com os de Dias et al. ${ }^{8}$ que demonstraram que indivíduos com maior aptidão cardiorrespiratória apresentavam melhor desempenho na execução de tarefas militares de cunho físico. Entretanto, nenhuma das medidas de desempenho de tiro analisadas apresentaram correlação com o condicionamento cardiorrespiratório. Uma possível explicação é a homogeneidade do condicionamento cardiorrespiratório da amostra que apresentou uma variação inferior a 9\%. Similarmente aos achados do presente estudo, Evans et al. ${ }^{22}$ não encontraram correlação entre o $\mathrm{VO}_{2 \text { pico, }}$ tempo até a exaustão em teste de esforço ou desempenho no teste de aptidão militar em nenhum dos parâmetros de tiro (acertos, erros e área de tiro) em soldados do exército.

Em estudo recente, Mon et al. ${ }^{2}$ avaliaram em atiradores sênior a associação entre o desempenho no tiro e a força muscular dos flexores dos dedos e adutores do ombro, assim como outras variáveis como idade, massa corporal, estatura, IMC, experiência com tiro e tempo semanal de treino. As únicas correlações significantes foram encontradas com a força muscular dos flexores dos dedos, a experiência em anos de treino e tempo semanal de treino. No referido estudo não foi analisada a aptidão cardiorrespiratória, o que não permite estabelecer comparações com o presente estudo.
Além disso, devemos considerar que o desempenho no tiro não é determinado apenas por variáveis físicas. As respostas neurais ao tiro ou a influência destas sobre o seu desempenho é um aspecto menos explorado. Nesse sentido, uma maior frequência elétrica cerebral de teta $(4-8 \mathrm{~Hz})$ tem sido associada a processos de atenção e melhor performance em tarefas de precisão, entretanto o esforço físico (cinco tiros de seis minutos em alta intensidade) não modificou a sua ocorrência em atletas treinados e não treinados em tiro, assim como também não modificou o desempenho ${ }^{18}$. Adicionalmente, Xu e Inzlicht ${ }^{23}$ mostraram que quando os indivíduos atiram erroneamente em uma alvo apresentam uma resposta neural associada ao erro maior que quando atiram corretamente. Interessantemente, essas respostas neurais associadas ao erro foram correlacionadas positivamente com a habilidade de atirar em alvos armados e não atirar em alvos desarmados. Infelizmente, essas respostas não foram mensuradas no presente estudo.

\section{CONCLUSÃO}

Os resultados do presente estudo demonstram que o esforço físico realizado previamente a execução de disparos não afeta o desempenho de tiro de policiais militares. Além disso, o nível de aptidão aeróbica não apresenta correlação com o desempenho de tiro de policiais militares, contudo, é importante para o desempenho físico realizado previamente aos disparos.

\section{AGRADECIMENTOS}

Os autores agradecem aos voluntários pelo tempo e energia despendido tornando esse estudo possível. Agradecemos também a Polícia Militar do Rio Grande do Norte pela colaboração nos cedendo seu espaço.

Todos os autores declararam não haver qualquer potencial conflito de interesses referente a este artigo.

CONTRIBUIÇÕES DE AUTORES: Cada autor contribuiu individual e significativamente para o desenvolvimento do manuscrito. LINN (0000-0003-1036-2649)*, DGSM (00000002-8426-0488)* e PMDA (0000-0003-4884-0730** contribuíram substancialmente na concepção, aquisição, análise, interpretação dos dados e redação do trabalho. LFFJ (0000-0003-4546-3079)*, AIF (0000-0003-1095-7845)*, SKAS (0000-0002-6884-7860)*, HME (0000-0003-3786-4999)* e AHO (0000-0002-8995-1392)* participaram na discussão dos resultados, revisão crítica do conteúdo do trabalho e redação. Todos os autores revisaram e aprovaram a versão final do manuscrito. *ORCID (Open Researcher and Contributor ID).

\section{REFERÊNCIAS}

1. Taverniers J, De Boeck P. Force-on-Force handgun practice: an intra-individualexploration of stress effects, biomarker regulation, and behavioral changes. Hum Factors. 2014;56(2):403-13.

2. Mon D, Zakynthinaki MS, Cordente CA, Antón AJ, Rodríguez BR, Jiménez DL. Finger flexor force influences performance in senior male air pistol olympic shooting. PLoS One. 2015;10(6):e0129862.

3. Oliveira Neto W, Guedes SPL. Esporte e patrimônio cultural: o tiro eas sociedades de atiradores em São Bento do Sul no início do século 21. In: Anuário do I Seminário Internacional História do Tempo Presente, Florinópolis; 2011.

4. Strahler J, Ziegert T. Psychobiological stress response to a simulated school shooting in police officers. Psychoneuroendocrinology. 2015;51:80-91.

5. Kayihan G, Ersöz G, Özkan A, Koz M. Relationship between efficiency ofpistol shooting and selected physical-physiological parameters of police. Polic An Int J Police Strateg Manag. 2013;36(4):819-32.

6. Souza MG, Momesso CM, Romanholo RA. Estresse e condicionamento físico: a influência na performance dos policiais do grupo de operações especiais de Cacoal/RO. Rev Bras Prescr Fisiol Exerc. 2011;5:21-6.

7. Brown MJ, Tandy RD, Wulf G, Young JC. The effect of acute exercise on pistolshooting performance of police officers. Motor Control. 2013;17(3):273-82.

8. Dias AC, Dantas EHM, Moreira SB, Furtado da Silva V. A relação entre onível de condicionamento aeróbico, execução de uma pista de obstáculose o rendimento em um teste de tiro. Rev Bras Med Esporte.2005;11(6):341-6

9. Thompson AG, Swain DP, Branch JD, Spina RJ, Grieco CR. Autonomic response totactical pistol performance measured by heart rate variability. J Strength Cond Res. 2015;29(4):926-33

10. Dadswell CE, Payton C, Holmes P, Burden A. Biomechanical analysis of thechange in pistol shooting format in modern pentathlon. J Sports Sci.2013;31(12):1294-301

11. Lakie M. The influence of muscle tremor on shooting performance. Exp Physiol. 2010;95(3):441-50.

12. Nibbeling N, Oudejans RR, Ubink EM, Daanen HA. The effects of anxiety and exercise-induced fatigue on shooting accuracy and cognitive performance ininfantry soldiers. Ergonomics. 2014;57(9):1366-79.

13. Tremayne P, Barry RJ. Elite pistol shooters: physiological patterning of best vs. worst shots. Int J Psychophysiol. 2001:41(1):19-29.

14. Ayoob MF. Ayoob Files: the book. 1st. ed. USA: PoliceBookshelf; 1995

15. Grebot C, Groslambert A, Pernin JN, Burtheret A, Rouillon JD. Effects ofexercise on perceptual estimation and short-term recall of shooting performancein a biathlon. Percept Mot Skills. 2003;97(3 Pt 2):1107-14.

16. Hoffman MD, Gilson PM, Westenburg TM, Spencer WA. Biathlon shootingperformance after exercise of different intensities. Int J Sports Med. 1992;13(3):270-3.

17. Borg GA. Psychophysical bases of perceived exertion. Med Sci Sports Exerc. 1982;14(5):377-81

18. Luchsinger $H$, Sandbakk $\varnothing$, Schubert M, Ettema G, Baumeister J. A comparison of frontal theta activity during shooting among biathletes and cross-country skiers before and after vigorous exercise. PLoSOne. 2016;11(3):e0150461.

19. Nibbeling N, Oudejans RR, Cañal-Bruland R, van der Wurff P, Daanen HA. Pursueor shoot? Effects of exercise-induced fatigue on the transition from running torifle shooting in a pursuit task. Ergonomics. 2013:56(12):1877-88.

20. Heyward VH. Advanced fitness assessment and exercise prescription. 7th. ed. Champaign, IL: Human Kinetics; 2006

21. Dadswell C, Payton C, Holmes P, Burden A. The effect of time constraints and running phases on combined event pistol shooting performance. J Sports Sci.2016;34(11):1044-50.

22. Evans RK, Scoville CR, Ito MA, Mello RP. Upper body fatiguing exercise and shooting performance. Mi Med. 2003;168(6):451-6.

23. Xu X, Inzlicht M. Neurophysiological responses to gun-shooting errors. Int J Psychophysiol. 2015;95(3):247-53. 